\title{
Uma estratégia para selecionar vértices como candidatos a roteadores em uma árvore de Steiner
}

\author{
João Guilherme Martinez ${ }^{1}$, Rosiane de Freitas ${ }^{1}$, Altigran da Silva ${ }^{1}$, Fábio Protti ${ }^{2}$ \\ ${ }^{1}$ Instituto de Computação - Universidade Federal do Amazonas (UFAM) \\ Manaus - AM - Brasil \\ ${ }^{2}$ Instituto de Computação - Universidade Federal Fluminense (UFF) \\ Niterói - RJ - Brasil \\ \{joaogam,rosiane,alti\}@icomp.ufam.edu.br, fabiodic.uff.br
}

\begin{abstract}
The Steiner tree optimization problem in graphs is NP-hard, however, algorithms that use heuristics obtain results close to optimal solution in polynomial time. In this paper we present a new algorithm based on an exact enumerative algorithm from literature. The proposed heuristic selects vertices as candidates to be routers in the Steiner tree.
\end{abstract}

Resumo. O problema da árvore de Steiner em grafos é NP-difícil, no entanto, algoritmos que fazem uso de heurísticas conseguem obter resultados próximos da solução ótima em tempo polinomial. Neste trabalho apresentamos um novo algoritmo baseado em um algoritmo exato enumerativo da literatura. A heurística proposta seleciona vértices como candidatos a serem roteadores na árvore de Steiner.

\section{Introdução}

Considere um grafo $G=(V(G), E(G))$ onde $V(G)$ representa o conjunto de vértices do grafo e $E(G)$ o conjunto de suas arestas. Dado um subconjunto $W$ de vértices de $V(G)$, uma árvore de Steiner consiste num subgrafo conexo $T$ de $G$ que contêm $W$ e cuja soma dos pesos das arestas de $E(T)$ seja mínimo. Os vértices do conjunto $W$ são chamados de terminais e os demais vértices de $V(G)$ utilizados para construir $T$ são chamados de vértices de Steiner. A versão de decisão do problema da árvore de Steiner em grafos é NP-Completo [Karp 1972], portanto não possui algoritmos polinomiais que encontrem a solução ótima, entretanto existem algoritmos na literatura que fazem uso de heurísticas e alcançam soluções próximas da ótima. O problema da árvore de Steiner possui aplicações práticas como o desenho de layouts de circuitos e o projeto de redes, além de outras variações do problema que surgem no campo prático.

Considere $n$ como o número de vértices em $V(G), m$ como o número de arestas em $E(G)$ e $k$ como o número de vértices terminais do conjunto $W$. A Tabela 1 apresenta alguns trabalhos relacionados publicados na literatura com a complexidade do algoritmo no pior caso e a razão de aproximação das soluções obtidas em relação à solução ótima, sendo alguns destes aproximativos ([Takahashi and Matsuyama 1980],[Wu et al. 1986],[Kou et al. 1981],[Zelikovsky 1993], [Robins and Zelikovsky 2000]) e outros exatos ([Dreyfus and Wagner 1971], [Vygen 2011],[Dourado et al. 2014],[Byrka et al. 2013],[Pajor et al. 2014]). 


\begin{tabular}{|l|l|c|c|l|}
\hline Título & Autores & Ano & Complexidade & $\begin{array}{l}\text { Razão } \\
\text { Aproximação }\end{array}$ \\
\hline $\begin{array}{l}\text { The Steiner problem in } \\
\text { graphs }\end{array}$ & $\begin{array}{l}\text { Dreyfus e Wag- } \\
\text { ner }\end{array}$ & 1971 & $O\left(n^{3}+n^{2} 2^{k-1}+n 3^{k-1}\right)$ & 1 \\
\hline $\begin{array}{l}\text { An approximate solution for } \\
\text { the steiner problem in graphs }\end{array}$ & $\begin{array}{l}\text { Takahashi e Mat- } \\
\text { suyama }\end{array}$ & 1980 & $O\left(k n^{2}\right)$ & 2 \\
\hline $\begin{array}{l}\text { A fast algorithm for Steiner } \\
\text { trees }\end{array}$ & $\begin{array}{l}\text { Kou, Markowsky } \\
\text { e Berman }\end{array}$ & 1981 & $O\left(k n^{2}\right)$ & $2(1-1 / l)^{\mathrm{a}}$ \\
\hline $\begin{array}{l}\text { A faster approximation algo- } \\
\text { rithm for the Steiner problem } \\
\text { in graphs }\end{array}$ & $\begin{array}{l}\text { Wu, Widmayer e } \\
\text { Wong }\end{array}$ & 1986 & $O(m \log n)$ & $2(1-1 / l)^{\mathrm{a}}$ \\
\hline $\begin{array}{l}\text { An 11/6-approximation algo- } \\
\text { rithm for the network steiner } \\
\text { problem }\end{array}$ & Zelikovsky & 1993 & $O\left(n m+k^{4}\right)$ & 1.833 \\
\hline $\begin{array}{l}\text { Improved Steiner Tree Ap- } \\
\text { proximation in Graphs }\end{array}$ & $\begin{array}{l}\text { Robins e Zeli- } \\
\text { kovsky }\end{array}$ & 2000 & & 1.55 \\
\hline $\begin{array}{l}\text { Faster algorithm for optimum } \\
\text { Steiner trees }\end{array}$ & Vygen & 2011 & $O\left(n k 2^{k+\log _{2} k \log _{2} n}\right)$ & 1 \\
\hline $\begin{array}{l}\text { Steiner Tree Approximation } \\
\text { via Iterative Randomized } \\
\text { Rounding }\end{array}$ & $\begin{array}{l}\text { Byrka, Grandoni, } \\
\text { Rothvoss e Sanità }\end{array}$ & 2013 & indefinido & 1.38 \\
\hline $\begin{array}{l}\text { Algorithmic aspects of Stei- } \\
\text { ner convexity and enumera- } \\
\text { tion of Steiner trees }\end{array}$ & $\begin{array}{l}\text { Dourado, Oli- } \\
\text { veira e Protti }\end{array}$ & 2014 & $O\left(n^{2}(n+m)+n^{k-2}+n \alpha\right)^{\mathrm{b}}$ & 1 \\
\hline $\begin{array}{l}\text { A Robust and Scalable Algo- } \\
\text { rithm for the Steiner Problem } \\
\text { in Graphs }\end{array}$ & $\begin{array}{l}\text { Pajor, Uchoa e } \\
\text { Werneck }\end{array}$ & 2018 & $O(m(m i n\{n k, m\}))$ & indefinido \\
\hline
\end{tabular}

Tabela 1. Algoritmos conhecidos na literatura para árvore de Steiner.

Na seção 2 são mostrados os conceitos preliminares para o funcionamento do algoritmo, o pseudo-código e a complexidade no pior caso. Na seção 3 são apresentados os resultados dos testes computacionais e na seção 4 são feitas as considerações finais.

\section{Descrição do Algoritmo}

O trabalho de Dourado et al. (2014) propõe um algoritmo exato enumerativo para obter as árvores de Steiner de um dado grafo em tempo exponencial para o número de terminais $k$. Nos conceitos preliminares, os autores explicam que os vértices de Steiner podem ser de dois tipos: linkers e roteadores. Os linkers são vértices de grau igual a 2 e os roteadores devem possuir sempre grau maior que 2. A partir disso, provam que em uma árvore de Steiner, sempre existe no máximo somente $k-2$ roteadores e que todo roteador ou terminal possui um caminho que é mínimo em $G$ até outro roteador ou terminal. Os vértices que formam esses caminhos são os linkers, por isso sempre possuem grau igual a 2 .

No algoritmo proposto pelos autores, são explorados todos os possíveis subconjuntos de roteadores que fazem parte da solução ótima, portanto consideram todos os subconjuntos com 0 a $k-2$ vértices como roteadores. Neste ponto o algoritmo torna-se exponencial na ordem de $O\left(n^{k-2}\right)$.

A heurística proposta neste trabalho consiste em analisar todos os vértices nãoterminais e usá-los como roteadores, um por vez, e analisar aquele que melhora a qualidade da última melhor solução obtida. No entanto esse procedimento só é executado até no máximo $k-2$ vezes, pois esta é a quantidade máxima de roteadores em uma árvore de Steiner mínima ótima. O Algoritmo 1 mostra o pseudo-código do algoritmo heurístico proposto.

\footnotetext{
${ }^{\mathrm{a}} l$ representa o número de folhas em uma árvore de Steiner ótima.

${ }^{\mathrm{b}} \alpha$ representa o número de árvores de Steiner ótimas enumeradas.
} 
Assim como no algoritmo exato [Dourado et al. 2014], a ideia geral consiste em utilizar uma matriz $d_{G}$ com as distâncias dos caminhos mínimos entre todos os vértices do grafo $G$ e construir templates, que são árvores geradoras mínimas de grafos completos $G_{R W}$ formados com os terminais e os roteadores, utilizando as distâncias em $d_{G}$ para os pesos das arestas.

A complexidade geral do algoritmo fica definida a seguir: para encontrar as distâncias $d_{G}$ é $O\left(n^{3}\right)$. A iteração principal do algoritmo é da ordem de $O\left(n k^{3}\right)$ devido ao algoritmo para gerar uma árvore geradora mínima no grafo completo $G_{R W}$. Portanto a complexidade total do algoritmo é $O\left(n^{3}+n k^{3}\right)$.

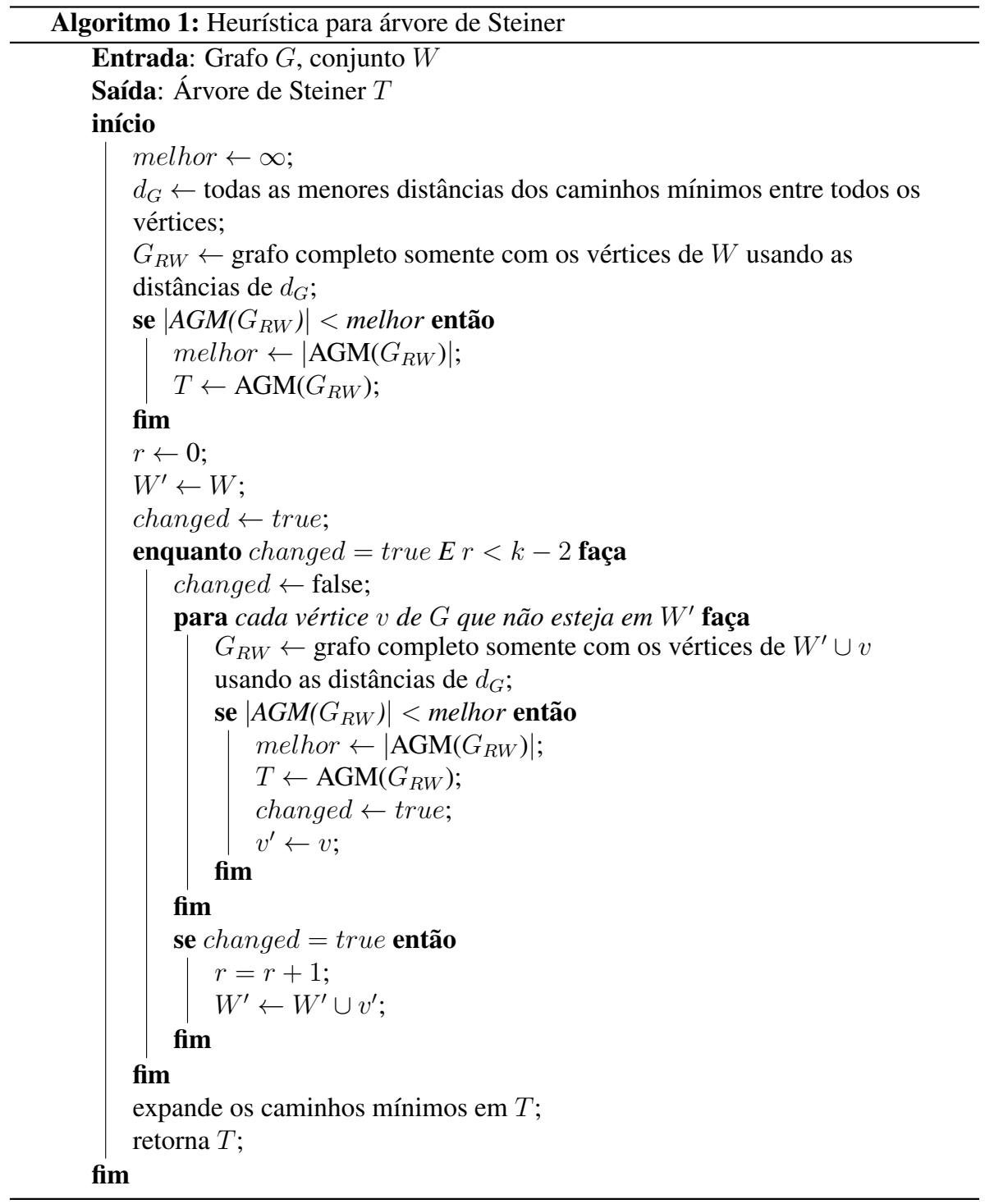

\section{Experimentos Computacionais}

A Tabela 2 apresenta os resultados dos testes computacionais realizados com as bases de teste de um benchmark conhecido ${ }^{c}$. As árvores encontradas pelo algoritmo foram comparadas com as soluções ótimas esperadas das instâncias em relação ao grau de

\footnotetext{
${ }^{\mathrm{c}}$ Bases de testes disponíveis em: http://steinlib.zib.de/testset.php
} 
aproximação. Para os experimentos foram utilizadas somente instâncias em que a solução ótima é conhecida.

\begin{tabular}{l|l|l|l|l|l|l} 
& $\begin{array}{l}\text { Esparsos } \\
\text { com pesos } \\
\text { aleatórios }\end{array}$ & $\begin{array}{l}\text { Completos } \\
\text { com pesos } \\
\text { aleatórios }\end{array}$ & $\begin{array}{l}\text { Esparsos } \\
\text { com pesos } \\
\text { euclidia- } \\
\text { nos }\end{array}$ & $\begin{array}{l}\text { Completos } \\
\text { com pesos } \\
\text { euclidia- } \\
\text { nos }\end{array}$ & $\begin{array}{l}\text { Esparsos } \\
\text { com pesos } \\
\text { incidentes }\end{array}$ & $\begin{array}{l}\text { TOTAL } \\
\text { Instâncias testadas }\end{array}$ \\
\hline Soluções ótimas encontradas & 60 & 27 & 14 & 14 & 395 & 514 \\
\hline Porcentagem de ótimas encontradas & $51,67 \%$ & $85,19 \%$ & $100 \%$ & $85,71 \%$ & $32,91 \%$ & $41,25 \%$ \\
\hline Média de aproximação & 1,01 & 1,00 & 1,00 & 1,00 & 1,01 & 1,01 \\
\hline Pior aproximação encontrada & 1,09 & 1,04 & 1,00 & 1,00 & 1,12 & 1,12
\end{tabular}

Tabela 2. Qualidade das soluções encontradas utilizando a heurística proposta.

\section{Considerações Finais}

A heurística proposta apresenta bons resultados em termos de qualidade da solução obtida em tempo $O\left(n^{3}+n k^{3}\right)$ e é de fácil compreensão e implementação. Melhorar os critérios de escolha dos vértices roteadores, obter a razão de aproximação do algoritmo e fazer um estudo comparativo com outras heurísticas são atividades para trabalhos futuros.

\section{Referências}

Byrka, J., Grandoni, F., Rothvoss, T., and Sanità, L. (2013). Steiner tree approximation via iterative randomized rounding. J. ACM, 60(1):6:1-6:33.

Dourado, M., Oliveira, R., and Protti, F. (2014). Algorithmic aspects of steiner convexity and enumeration of steiner trees. Annals of Operations Research, 223(1):155-171.

Dreyfus, S. E. and Wagner, R. A. (1971). The steiner problem in graphs. Networks, 1(3):195-207.

Karp, R. M. (1972). Reducibility among Combinatorial Problems, pages 85-103. Springer US, Boston, MA.

Kou, L., Markowsky, G., and Berman, L. (1981). A fast algorithm for steiner trees. Acta Informatica, 15(2):141-145.

Pajor, T., Uchoa, E., and Werneck, R. F. (2014). A robust and scalable algorithm for the steiner problem in graphs. CoRR, abs/1412.2787.

Robins, G. and Zelikovsky, A. (2000). Improved steiner tree approximation in graphs. In Proceedings of the Eleventh Annual ACM-SIAM Symposium on Discrete Algorithms, SODA '00, pages 770-779, Philadelphia, PA, USA. Society for Industrial and Applied Mathematics.

Takahashi, H. and Matsuyama, A. (1980). An approximate solution for the steiner problem in graphs. In Math. Jap, pages 24:573-577.

Vygen, J. (2011). Faster algorithm for optimum steiner trees. Information Processing Letters, 111(21):1075 - 1079.

Wu, Y. F., Widmayer, P., and Wong, C. K. (1986). A faster approximation algorithm for the steiner problem in graphs. Acta Inf., 23(2):223-229.

Zelikovsky, A. Z. (1993). An 11/6-approximation algorithm for the network steiner problem. Algorithmica, 9(5):463-470. 